$\begin{array}{ll}\text { Italique } & \text { Italique } \\ \text { Poésie italienne de la Renaissance }\end{array}$

V | 2002

Varia

\title{
«Del fuggir la moltitudine ». Néoplatonisme et scepticisme dans le Malpiglio secondo du Tasse
}

\section{Matteo Residori}

\section{OpenEdition}

Journals

Édition électronique

URL : http://journals.openedition.org/italique/150

DOI : $10.4000 /$ italique. 150

ISSN : 1663-4438

Éditeur

Librairie Droz

Édition imprimée

Date de publication : 31 décembre 2002

Pagination : 93-108

ISBN : 2-600-00826-8

ISSN : 1423-3983

Référence électronique

Matteo Residori, « «Del fuggir la moltitudine ». Néoplatonisme et scepticisme dans le Malpiglio secondo du Tasse », Italique [En ligne], V | 2002, mis en ligne le 06 octobre 2009, consulté le 01 mai 2019. URL http://journals.openedition.org/italique/150 ; DOI : 10.4000/italique.150

(c) Tous droits réservés 


$$
\text { MATTEO RESIDOR I }
$$

«DEL FUGGIR LA MOLTITUDINE».

N É OPLA T ONISME ET SCEPTICISM E

DANS LE MALPIGLIO SECONDO

D U T A S E 


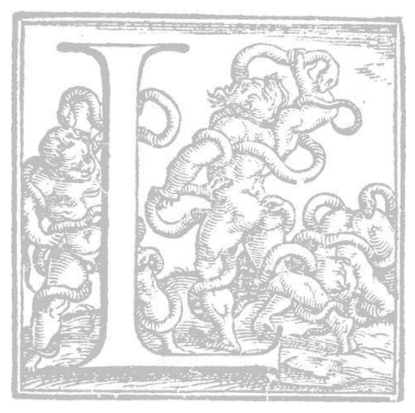

A composition, en $1584-1585$, du dyptique de dialogues consacré à Vincenzo et à Giovanlorenzo Malpiglio semble destinée à illustrer la double vocation de l'cuvre dialogique $d u$ Tasse, telle que l'auteur la définit à la même époque dans son Discours sur le dialogue. ${ }^{\mathrm{I}}$ Le Malpiglio overo de la corte, qui a pour protagoniste le gentilhomme lucquois Vincenzo Malpiglio, trésorier du duc de Ferrare, est un exemple parfait de dialogue «civil ou moral», portant sur les problèmes éthiques et politiques de la vie de cour. Le Malpiglio secondo overo del fuggir la moltitudine semble pencher plutôt du côté du genre «spéculatif», qui a pour objet les questions concernant "la science et la vérité». Mais cette définition n'est pas assez précise. En mettant en scène le fils de Vincenzo, Giovanlorenzo, jeune bomme brillant qui aspire à l'otium littéraire et philosophique, le Tasse propose dans le Malpiglio secondo une réflexion sur les fondements mêmes de l'activité spéculative, et, de ce fait, sur la légitimité de sa propre entreprise littéraire. Dans la lecture du dialogue il faudra tenir compte de cette dimension réflexive, tout en essayant d'en préciser les circonstances et la portée réelle. D'autre part, la complexité du parcours que le Tasse dessine dans le texte, qui compte parmi les plus ambigus des Dialogues, demande an lecteur de porter une attention particulière aux modèles qui en organisent la texture composite. C'est dans cette direction que nous avons orienté notre lecture, pour essayer de démonter la machine complexe $d u$ Malpiglio secondo et d'éclairer ainsi quelques uns de ses enjeux essentiels. ${ }^{2}$

Le titre du dialogue - del fuggir la moltitudine - semble annoncer la reprise anodine d'un lieu commun de la culture bumaniste: celui qui consiste à exalter la solitude contemplative en l'opposant à la dispersion et à la superficialité de la vie sociale. L'impression est confirmée par la première page du texte, qui présente la description du «studio» que le riche Vincenzo Malpiglio a fait construire pour son fils dans le palais familial, au cour de Ferrare. Ce lieu silencieux et confortable, dont la position élevée semble propice aux activités de l'esprit, rassemble dans un ordre parfait tous les instruments du loisir lettré:

Il signor Vincenzo Malpiglio è gentiluomo in cui le ricchezze non sono impedimento de la virtù, come in molti suole avenire, ma ornamento, come in pochi suoi pari: laonde non solo procura che sia adornato l'animo del figliuolo, ma lo studio ancora, il quale è ne la più alta parte de la casa, posta ne la più frequentata de la città. Quivi essendo io montato per una lunga scala, già stanco, mi posi a sedere sovra una sedia e sovra un coscino di cuoio, il quale ne la caldissima 
stagione porgea gratissimo ristoro a l'affaticate membra; e risguardando intorno, non faceva motto, sì perché 'l ragionare m'era impedito da l'anelito, sì per la novità de le cose vedute, le quali traevano gli occhi a rimirare. Perciò ch'a la prima vista mi si parò dinanzi una grandissima quantità di bei libri di tutte le lingue, di tutte le scienze, ben ligati con fette di seta; e molti quadri di pittura assai vaghi, e alcune vaghe tavole di geografie, ne le quali diligentemente sono descritti vari paesi, e alcuni globi o palle, fatte ad imagin del mondo con la descrizione del cielo e de la terra; e altre palle di marmo di varî colori, e varî cristalli da ristorar la vista e vari instrumenti di musica; altri da osservar l'altezza del polo, altri per tutti gli usi de l'astrologia e de la geometria; e tutte queste cose erano in guisa disposte ch'altrettanto meritava d'esser lodato l'ordine quanto la vaghezza. ${ }^{3}$

Sur les étagères s'alignent des livres magnifiquement reliés en soie, «dans toutes les langues et sur toutes les sciences»; les murs sont décorés de tableaux; des mappemondes et des cartes geographiques reproduisent l'image "du ciel et de la terre». Le regard fatigué par la lecture trouve à se soulager en se posant sur des cristaux bariolés et des sphères de marbre, tandis que plusieurs instruments de musique sont là, à portée de main, pour détendre l'esprit. D'autres instruments permettent, enfin, les calculs géométriques et astronomiques. Cette description traduit en image un idéal de culture fondé sur l'universalité du savoir, sur la structuration harmonieuse et cobérente des connaissances et sur l'equilibre parfait entre étude et plaisir. Le «studio» est un microcosme non moins admirable pour la beauté («vaghezza») que pour l'ordre («ordine») de ses éléments. Il présente une synthèse des connaissances et des activités de l'esprit, qui allie de manière exemplaire la variété à l'unité. ${ }^{4}$

Le lieu où le jeune Giovanlorenzo a choisi de «fuir la multitude» des hommes semble donc extrêmement propice au recueillement et à la paix de l'esprit. Le dialogue se chargera de prouver le contraire, en dénonçant la fragilité de cet idéal de culture. L'aspirant bumaniste reçoit la visite de l" Étranger Napolitain», masque habituel du Tasse dans les Dialogues, qui va lui démontrer que la solitude physique ne suffit pas à exclure la «multitude», et que les livres de cette silencieuse bibliothèque constituent une foule aussi nombreuse et aussi bruyante que celle qui occupe les rues de la ville et les salons de la cour.

En effet, l'«Étranger» interprète le mot «moltitudine» en sens abstrait et philosophique: il ne s'agit pas, à ses yeux, d'une 'multitude' qui s'opposerait à la solitude, mais d'une 'multiplicité' qui menace, de l'intérieur non moins que de l'extérieur de l'homme, cette 'unité' qui est la condition nécessaire de l'equilibre spirituel. La question éthique traditionnelle - faut-il choisir la solitude ou la vie sociale? - cède ainsi la place à une enquête philosophique plus vaste autour des deux notions d'unité et de multiplicité, qui occupent une place centrale dans toute l'cuvre du Tasse. 
Cette opposition est évidemment platonicienne. Mais il est possibile de déterminer plus précisément son origine, en étudiant de près les sources du dialogue. Parmi les livres ayant appartenu au Tasse, qui sont conservés en grande partie à la Bibliothèque Vaticane, se trouve un petit recueil d'écrits néoplatoniciens, choisis et traduits en latin par Marsile Ficin, qui fut publié par Alde Manuce en 1497 et connut un certain succès tout au long du XVT siècle. ${ }^{5}$ Ce recueil comprend, sous le titre De anima et daemone, des extraits du vaste commentaire que le philosophe néoplatonicien Proclus consacra au premier Alcibiade de Platon, un texte très important pour la transmission des idées néoplatoniciennes. Un de ces extraits, que Ficin intitule Multi gradus per quos a multitudine ad unum adscendimus, est la source directe $d u$ Malpiglio du Tasse, qui en traduit à la lettre quelques passages et emprunte à Proclus, le titre et le schéma d'ensemble de son dialogue. Voici les premières lignes du texte, qui présentent le parcours qui conduisant graduellement de la multitude à la parfaite unité du savoir:

Plato et Pithagoras iubent fugere multitudinem, ut veritatem simplicissimam consequamur. Multitudinem quidem exterioris populi, nos multifariam distrahentis atque fallentis; interioris quoque populi multitudinem multo magis: haec enim magis distrahit atque fallit. Primam ergo fugito variam affectuum multitudinem. Secundo sensuum. Tertio imaginationum. Quarto opinionum inde pendentium. Omnis enim multitudo iam dicta tam in se diversa est, ut alia aliis in qualibet contraria sint. Unde praestat ad scientias se conferre. In quibus multitudo iam contrarietatem habet nullam. ${ }^{6}$

Ce parcours philosophique comprend plusieurs étapes. Il faut d'abord se soustraire au domaine des emotions (affectus), des sensations (sensus), des images (imagines) et des opinions (opiniones), où règnent une multiplicité contradictorie et une angoissante instabilité. L'bomme soucieux de son bonheur spirituel doit plutôt se consacrer à la science (scientia), qui est encore marquée par la multiplicité, mais sait organiser ses multiples éléments en un ensemble cohérent et hiérarchisé. Le passage où Proclus expose ce concept est traduit fidèlement par le Tasse:

Nam etsi affectus affectibus contrarii sint, sensus quoque sensibus, imaginationes imaginationibus, opiniones opinionibus nulla tamen scientia scientiae contraria reperitur [...] Tanto enim abest quod invicem contraria sint, ut etiam notio notioni serviat, scientiaeque inferiores omnes superiori ministrent, habeantque sua $\mathrm{ab}$ illis exordia. In hac igitur propositionum notionumque multitudine congregemus in unum scientiarum quoque numerum uno quodam vinculo connectamus. $^{7}$ 
Noi dicemmo nel principio che gli affetti son contrari a gli affetti, l'imagini a l'imagini, l'opinioni a l'opinioni; ma fra le scienze non è contrarietà, perché la scienza inferiore serve a la superiore, quasi ministra, e piglia da lei i princìpi. ${ }^{8}$

Cette étape, très importante, n'est pourtant pas la dernière. Après s'être formé à la scientia, le vrai philosophe doit se tourner vers l'intellectus, connaissance intuitive de l'unité intelligible, qui est totalement transcendante:

Post vero scientiam eiusdem studium expedit iam deponere compositiones et divisiones multiformesque discursus, atque illinc ad intellectualem vitam simplicesque intuitus et perceptiones eius ascendere. Scientia enim non est summus cognitionum apex. Sed super eam est intellectus, non intellectum inquam dumtaxat ab anima separatum, sed ipsam quoque illustrationem animae illinc infusam, de quo et Aristoteles ait intellectum esset, quo terminos cognoscimus. Atque Timaeus hunc ipsum inquit in nullo unquam alio praeterquam in anima fieri. Ad intellectum igitur hunc ascendentes una cum intelligibilem essentiam contemplemur. ${ }^{\text {? }}$

Ma volendo seguirle e fuggir quanto si può la moltitudine e la contrarietà che insieme contiene, fa di mestieri che depognamo le composizioni e le divisioni e $\mathrm{i}$ vari discorsi, e ascendiamo a la contemplazione e al conoscimento e quasi a la semplice vista del vero; perché la scienza non è la somma cima de la cognizione, ma sovra lei è l'intelletto; né solamente quel ch'è ne l'animo seperato, ma quello co 'l quale dice Aristotele ch'intendiamo i termini, il qual Timeo aferma che non è in alcuno altro che ne l'anima. A questo intelletto adunque ascendendo, insieme contempleremo l'intelligibile essenza. ${ }^{\text {Io }}$

La présence de ce texte parmi les sources du dialogue n'a rien de très étonnant, de nombreux travaux ayant déjà démontré l'importance du néoplatonisme dans la culture du Tasse. ${ }^{\text {I I }}$ D'autre part, ces mêmes travaux ont montré que le Tasse recourt souvent à la pure et simple traduction de ses sources. Quoiqu'elle ait parfois été considérée comme un symptome de lassitude intellectuelle, cette habitude semble plutôt révéler une relation particulièrement étroite entre lecture et écriture: les Dialogues sont aussi un recueil d'excerpta, d'extraits de lecture, un moyen de fixer sur la page les connaissances acquises à travers des lectures diverses, qui deviennent le point de départ de nouveaux textes.

Le Malpiglio emprunte à Proclus son schéma logique, à savoir la description du parcours graduel conduisant de la multiplicité à l'unité, mais avec des différences importantes. D'abord, le Tasse applique ce schéma plus particulièrement au domaine culturel: la conformité à l'idéal d'unité permet de juger la valeur des livres qui composent l'admirable bibliothèque du jeune Malpiglio. 
L'autre différence consiste dans la place que le Tasse attribue à la scientia, c'est-à-dire à l'ensemble des connaissances scientifiques et philosophiques héritées de l'antiquité: loin d'être considérée comme un patrimoine unitaire et cohérent, elle fait l'objet d'une critique radicale, on le verra, en raison précisément de son manque de cohérence.

La première partie du dialogue constitue un étonnant morceau de critique littéraire. Pour vérifier si la vie solitaire et studieuse est vraiment exempte de toute multiplicité, l'Étranger Napolitain invite d'abord son jeune ami à relire avec lui les livres de sa bibliothèque qui lui sont les plus chers: les cuvres de poésie. Or, quelques citations de Pétrarque suffisent à montrer que ces livres, au lieu de rendre plus agréable la solitude, la détruisent complètement et lui substituent une foule d'émotions, d'images et d'opinions différentes.

Si la poésie multiplie les émotions, c'est parce qu'elle engendre dans le lecteur l'adhésion émotive aux passions d'un auteur extremement changeant, déconcertant Protée qui passe sans cesse des larmes au rire, de l'euphorie au deséspoir, du désir charnel à la contrition. La multiplication des images provient du powvoir qu'a la poésie de créer de veritables illusions optiques, en mettant les choses «sous les yeux du lecteur», selon la définition classique de l'enàrgheia: le jeune Malpiglio a beau s'enfermer dans son «studio», la lecture de Pétrarque fait déflier devant ses yeux des douces collines, des sources d'eau pure, des visages de femmes. Enfin, la poésie multiplie les opinions, parce que les poètes semblent ignorer ou mépriser le principe de non contradiction: Pétrarque peut avoir, sur le même sujet, des opinions très diffèrentes; et la pluralité des auteurs et des cuvres ne peut que multiplier à l'infini cette variété contradictoire. La lecture des livres de poésie est donc énergiquement déconseillée à celui qui cherche à «fuir la multitude»; et l'Étranger Napolitain finit par inclure dans sa condamnation, sans s'embarrasser d'autres démonstrations, les poètes épiques, les tragiques et les orateurs, c'est-à-dire la littérature toute entière.

Après avoir constaté le caractère foncièrement multiple de la poésie, les deux personnages du dialogue se tournent, suivant le schéma néoplatonicien, vers le vaste domaine des «sciences». Cette partie du texte, qui occupe à peu près les deux tiers de son extension, est sans doute la plus surprenante. L'exploration de l'énorme héritage philosophique de l'antiquité se fait à travers la métaphore filée de la navigation sur une mer agitée à la recherche d'un port tranquille. Cette métaphore, certes traditionnelle, a une importance toute particulière dans l'oeuvre du Tasse, qui dès sa jeunesse en fait l'image obsédante de son désarroi existentiel. ${ }^{12}$ Dans notre dialogue, elle prend une ampleur considérable et devient une sorte de schéma mnémotechnique destiné à rendre visibile l'organisation des sciences à travers un système minutieux de correspondances entre les parties de l'encyclopédie et les éléments d'un paysage marin. ${ }^{{ }^{3}}$ 
Le navire de l'intelligence longe une côte escarpée sur laquelle s'ouvrent, à droite et à gauche d'un promontoire inaccessible, plusieurs ports: les uns représentent les idées de Platon et de son école, les autres les théories d'Aristote et des péripatéticiens. Les ports sont plus ou moins vastes selon l'importance du courant philosophique qu'ils représentent; en outre, à l'intérieur de certains ports se trouvent des baies qui indiquent les articulations ultérieures de chaque école. Le navire explore toute la côte à la recherche d'un port tranquille, mais trouve partout une mer qu'agitent les vents des disputes, des contradictions, des incobérences. Contrairement à ce que pensait Proclus, le domaine des sciences semble donc réfractaire à toute forme d'barmonie unificatrice et de conciliation dialectique.

Il faut maintenant observer de plus près cette section du dialogue. L'extrait suivant, concernant les théories de l'âme, donne une idée assez précise de la stratégie mise en place par l'auteur; la longueur de la citation vise à respecter les intentions stylistiques du texte, qui exploite systematiquement la figure de l'accumulation pour produire un effet de saturation:

Né minor discordia è ne la anima di quel che sia ne la generazione [...] Molti di coloro che vissero inanzi ad Aristotele ebbero opinione che l'animo fosse quello che muove prima grandissimamente: perciò disse Democrito che l'animo è certo fuoco, perch'essendo infinito il numero de le figure e de' corpicciuoli che non possono esser divisi, egli stimò che l'animo fosse composto di quelle che son più ritonde, quali sogliono vedersi ne l'aria e ne' raggi quando il sole entra per le fenestre [...] ma Empedocle riputò che l'animo fosse un componimento de quattro elementi e ch'egli vedesse la terra con la terra, l'acqua con l'acqua, l'aria con l'aria e 'l fuoco co 'l fuoco, con l'amore l'amore e la discordia con la discordia [...] Ma percioché l'animo par che sia quello c'ha forza di muovere e di conoscere, alcuni hanno congiunto insieme queste cose e detto che l'animo sia numero che si muova da se stesso. Diogene ancora, com'alcuni altri, pensò che l'animo fosse aere, il quale è principio sottilissimo oltre tutti gli altri, e per questa cagion disse ch'egli moveva e conosceva. Ma Eraclito stimò che fosse quel vapore del qual son fatte le cose tutte, e Alcmeone portò de l'animo la medesima opinione che gli altri, dicendo ch'egli era immortale e per questo s'assomigliava a le cose immortali: e quel che sempre muove a lui si conveniva. Ma fra coloro che sono più importuni alcuni dissero che ch'egli è l'acqua, peroch'il seme di tutte le cose è l'acqua percioché egli è umido; altri, fra' quali è Critio, pongono ch'egli sia il sangue: e in somma tutti gli elementi sono stati giudicati de la natura de l'anima [...] Altri vollero che l'animo fosse armonia o non senza armonia, ma tutti il diffiniscono o dal moto o dal senso o da l'incorporeo. Ma Aristotele, avendo riprovato l'opinione degli altri, adduce la sua, la quale è che l'anima sia la forma o l'atto o la perfezione del corpo naturale. ${ }^{\mathrm{I}}$ 
Comme on le voit, l'Étranger Napolitain dresse une simple liste des opinions des philosophes, et souligne moins ce qui les unit que ce qui les oppose. On ne trouve là aucune trace de biérarchie, aucune tentative de discussion ni de synthèse. Toutes les idées se valent, et aucune ne peut prétendre exprimer la vérité. Cette stratégie produit un effet de chaos, d'entassement stérile, de bruit discordant et confus.

L'exploration du patrimoine culturel de l'antiquité continue de cette façon pour une vingtaine de pages: les deux personnages prennent en considération, l'une après l'autre, les principales questions philosophique, et pour chacune ils recensent des dizaines de réponses différentes et contradictoires. $A$ la fin de ce parcours frustrant, la culture ancienne semble réduite à un tas de décombres informes et inutilisables. Les opinions des philosophes ne sont que les voix d'un débat interminable, qui ne laisse entrevoir aucune perspective d'unité. La multiplicité est dans ce domaine encore plus vertigineuse et plus déconcertante que dans la littérature.

Pour interpréter correctement cette stratégie, il faut parler encore une fois des sources du dialogue, de ses sources anciennes et de ses probables modèles modernes. Comme l'a montré Ettore Mazzali, le Malpiglio secondo dépend largement d'un texte de Plutarque, à savoir l'cuvre doxographique connue sous le titre latin De placitis philosophorum: ce répertoire d'ropinions des philosophes» fournit en effet la plupart des informations qui étoffent la section scientifique du dialogue. ${ }^{15}$ Mais le plus intéressant est que le Tasse transforme en stratégie stylistique ce qui était chez. Plutarque un trait formel anodin, voire une loi du genre: la simple juxtaposition des idées des philosophes. En passant du repertoire doxographique au dialogue, ces listes perdent leur fonction pratique originaire - comparable à celle d'un manuel - pour devenir l'expression formelle d'un savoir dramatiquement fragmenté. Les quelques interventions que le Tasse opère sur son modèle ancien sont destinées à accentuer cet effet: d'une part, à travers la syntaxe, les conjonctions et les adverbes, il s'efforce de transformer la juxtaposition en conflit; de l'autre, il tend à effacer la mince architecture logique du texte de Plutarque, en en supprimant les introductions et les synthèses et en allant jusqu' à bouleverser l'ordre des opinions citées. ${ }^{16}$

Comme pour beaucoup d'autres hommes de lettres de la Renaissance, la littérature doxographique est très importante pour le Tasse. Pendant la longue période de réclusion à Sainte-Anne, où il se plaint sans cesse de la pénurie de livres, les ceuvres de Plutarque et de Stobée lui tiennent lieu de bibliothèque. Elles viennent au secours de sa mémoire, qu'il sent de plus en plus incertaine, et l'aident à réaliser ses ambitions encyclopédiques, en lui permettant d'émailler sa prose et ses vers de citations érudites. ${ }^{17}$ Plus que nulle autre 
cuvre du Tasse, les Dialogues dépendent de ces sources, où ils puisent un matériau riche et multiforme: définitions, exemples, citations, résumés de théories philosophiques. Ces repertoires de la culture antique alimentent la «ariété» des Dialogues, que le Tasse développe parfois jusqu'à un éclectisme précieux et décoratif. Toutefois, les instruments de la dialectique ou une autorité plus forte que les autres viennent en général endiguer cette prolifération et la réduire à l'unité. ${ }^{\mathrm{I}}$

Le Malpiglio secondo pousse ce procédé juste un peu plus loin que d'autres dialogues, mais cela suffit pour transformer la variété en «multitude» non maîtrisée. Le Tasse se montre ici plus sensible à l'ambigüité statutaire de la doxographie, qui traite les idées philosophiques comme des 'opinions' réversibles et interchangeables, et risque ainsi de réduire la «science» à une forme de sophistique - ou de littérature. Il semblerait donc que le Tasse ait voulu explorer, dans le Malpiglio, le chaos qui menace les meticuleuses architéctures dialectiques de ses dialogues.

Restent à déterminer les motifs de cette étrange opération. Sagit-il d'un moment de crise dans le projet philosophique du Tasse? Ou plutôt d'une sorte de mise en scène cathartique, destinée à conjurer une fois pour toutes l'angoissante multiplicité qui menace la réalisation de ce projet? La saveur quelque peu 'borgesienne' du Malpiglio, voyage vertigineux à l'intérieur d'une bibliothèque, risque d'encourager les interprétations anachroniques, comme celle qui ferait de ce texte le document visionnaire d'une sorte d'indigestion livresque, consommée dans le sombre huis clos de la prison de Sainte-Anne. Si cette interprétation caricaturale frôle, sans le nommer, le spectre de la folie du Tasse, celle qu'a proposée récemment Giovanna Scianatico risque à l'inverse d'attribuer à l'auteur une trop grande clairvoyance historique. Il est difficile de croire, en effet, que le Malpiglio puisse décrire l'«effondrement de l'ancienne vision du monde, du cadre traditionnel des savoirs et de l'organisation de l'univers», ${ }^{19}$ lorsque tous les indices à notre disposition tendent à prouver au contraire que la culture scientifique du Tasse était parfaitement conventionnelle et bien protégée contre toute inquiétude 'copernicienne. ${ }^{20}$ En réalité, le désarroi culturel que le dialogue exprime est tout sauf moderne et révolutionnaire.

La stratégie mise en place dans la section scientifique du Malpiglio n'est pas sans rappeler celle qu'adoptent les représentants du "pyrrbonisme chrétien". Cette expression désigne un courant philosophique, minoritaire mais important dans la culture de la Renaissance, qui récupère le scepticisme radical de Sextus Empiricus pour dénoncer le caractère contradictoire et instable du savoir humain et lui opposer la solidité de la révélation chrétienne. ${ }^{2 \mathrm{I}}$ Parmi les représentants de cette tradition, qui culmine dans un texte célèbre et complexe comme l'Apologie de Raimond Sebond de Montaigne, le plus connu est 
sans doute Jean-François Pic de la Mirandole, qui publie en Is20 l'Examen vanitatis doctrinae gentium, immense traité recensant méthodiquement le patrimoine de la culture classique afin de démontrer son incobérence et de contester les ambitions de la raison bumaine.

Cette cuvre rencontra quelqeu notoriété au XVT siècle, ne serait-ce que par la radicalité de son attaque contre les ideaux culturels de l'bumanisme, ${ }^{22}$ de sorte qu'il n'est pas impossible que le Tasse l'ait connue. Quoi qu'il en soit, entre la technique polémique de l'Examen vanitatis et celle qu'adopte le Malpiglio secondo, ily a une affinité évidente. Une citation prise au hasard suffira à montrer que Jean-François Pic veut produire le même effet d' accumulation exaspérante que nous avons observé dans le dialogue du Tasse:

Iam nec maior in ipsa rei natura et essentia quam in numero et ordine consensus apparet. Alii igneos putarunt, alii chrystallinos, alii nullo pacto quatuor elementorum essentiae participantes, sed quintae cuiusdam naturae propriae, et ab inferioribus omnino seiunctae, cuius sententiae primus author prohibetur Aristoteles, ita ut calorem ipse in sese suaque forma non contineat, sed sua virtute calorem inferioribus corporibus impartiat. Democritus vero solem nullo modo calidam habere naturam affirmavit, authore Istromatis Plutarcho. At in stellis et planetis fortasse concordes putaveris: solem et lunam igneas nubes esse putavit Xenophanes [...] Thales vero terrestres censuit, et ex terra quoque illos fabricavit Anaximenes. Atqui nec in figura concors sententia, spherica esse Aristoteles voluit, et naturam quinti corporis habere; Heraclitus in scaphae speciem formatum solem maluit et lunam; nec defuere qui curruli rotae similia fecerint. Anaximenes latum esse solem et veluti aulea quaedam extentum affirmavit. Alii lunam eylinidis forma conditam statuerunt. Maluit Diogenes esse hederaceae frondis specie, et Philolaus Pythagoricus chrystallina, ex igne tamen qui in universo est splendorem assumere. Existimatum est a Platone plurimum quidem ignis habere solem et lunam, non tamen esse aliorum elementorum expertia. ${ }^{23}$

Si les traits sceptiques de l'écriture du Malpiglio sont évidents, le sens de cette stratégie et l'intention idéologique du dialogue continuent à ne pas être complètement clairs. Peut-on affirmer que le Tasse souscrit à cette polémique radicale contre la culture classique et contre l'orgueil intellectuel des hommes? Certainement pas. La conclusion du Malpiglio semble indiquer, malgré son caractère elliptique, que le 'scepticisme' du Tasse a plutôt une fonction éthique et politique.

L'échec par lequel se solde l'exploration des sciences à la recherche de l'unité pousse l'Étranger Napolitain et Giovanlorenzo Malpiglio à se tourner vers le dernière étape du parcours dessiné par Proclus: la contemplation. Dans le paysage métaphorique de cette aventure de la connaissance, l'unité transcen- 
dante est représentée par un Temple de la Concorde, situé sur le sommet d'une montagne qui surplombe la côte, à mi-chemin entre le port de Platon et le port d'Aristote. Ma l'Étranger Napolitain, ni son jeune ami ne se sentent en condition d'entreprendre une ascension si redoutable. Même s'ils admettent que seule la contemplation intellectuelle leur permettrait d'atteindre l'unité parfaite de la connaissance et la paix absolue qui l'accompagne, ils voient que cela demande une force d'esprit hors du commun et exigerait d'eux des sacrifices qu'il ne sont pas prêts à faire. Dans les toutes dernières répliques l'orientation du discours change donc brusquement et amène à une conclusion inattendue:

G. M. Questa fuga è solamente convenevole a gli uomini che vogliono esser molto più ch'uomini e poco meno ch'iddii; ma noi, che non vogliamo lasciare ogni azione, dove rifuggiremo?

F. N. Fuggite, quando che sia, da la solitudine a la moltitudine per il giovamento de la patria, e tutte le vostre fughe saranno onorate. ${ }^{24}$

La seule expérience intellectuelle digne de ce nom est la contemplation; mais la contemplation est une activité exigeante, digne d'hommes "qui veulent être plus que des hommes et presque des dieux», et exclut toute forme d'action et de sociabilité. Il ne reste donc qu'à abandonner les ambitions contemplatives et à quitter la solitude à la multitude, c'est-à-dire pour la vie sociale au service de la patrie. L'adoption d'un point de vue sceptique a donc la double fonction de contester les ambitions intellectuelles immodérées et de promouvoir l'action au détriment de la contemplation. Le jeune Giovanlorenzo est finalement invité à suivre les traces de son père, et le dialogue qui devait explorer un mode de vie différent de celui du courtisan se conclut par une exhortation à la vie de cour ce qui confere au dyptique sur les deux Malpiglio, in extremis et quelque peu inopinément, une plus profonde unité.

Quels sont les enjeux d'une telle conclusion? Pour les évaluer il est nécessaire de sortir de la fiction du dialogue pour entrer dans la biographie du Tasse, qui, en I585, se trouvait encore à l'hôpital de Sainte-Anne et essayait par tous les moyens d'en sortir. Dans ce contexte, il est tentant de lire l'exhortation finale du dialogue comme un soubait et une promesse: le soubait d'être à nouveau accueilli dans le port de la cour, plus tranquille que tous les ports philosophiques; la promesse d'abandonner un orgueil intellectuel stérile. Et l'on peut peut-être pousser l'interprétation jusqu'à voir dans la navigation sur la mer des sciences la métaphore d'autres expériences intellectuelles, de bien plus dangereuses aventures de l'esprit: celles qui avaient conduit le Tasse devant le père inquisiteur de Ferrare et n'étaient sans doute pas étrangères à son emprisonnement. Si cette bypothèse était juste, la théologie, complètement absente du dialogue, en serait en quelque sorte le protagoniste secret; et les vents 
qui agitent la mer de la multitude tireraient leur force effrayante d'une inquiètude religieuse qui n'ose plus dire son nom.

Dans l'Apologie de Raimond Sebond, Montaigne avait choisi le destin malheureux du Tasse pour illustrer l'imperceptible «voisinage» entre la «folie» et les "gaillardes élévations d'un esprit libre», et justifier ainsi son éloge de la modération intellectuelle. ${ }^{25}$ L'exploration 'sceptique' de la folie multiforme des sciences que le Tasse met en scène, avec la plus grande lucidité, quelques années plus tard, semble aboutir à la même conclusion pragmatique et désenchantée ce qui n'empêche pas le spectacle mouvant et vertigineux de la «multitude» d'exercer sur lui, et sur le lecteur, une étrange et persistante fascination.

Matteo Residori 
I. Torquato Tasso, Discours sur le dialogue, traduction de F. Vuilleumier, notes de G. Baldassarri, préface de N. Ordine, Paris, Les Belles Lettres, I992, pp. 70-71.

2. Cet article reproduit le texte d'une conférence prononcée le 6 mai 2002 à l'Université de Genève dans le cadre des travaux du "Groupe d'Étude sur le XVI ${ }^{\mathrm{e}}$ siècle". Je souhaite remercier les responsables du groupe, Olivier Pot, Giovanni Bardazzi et Richard Waswo, pour leur invitation et leur attention bienveillante, et Guglielmo Gorni, pour avoir accepté d'accueillir cet article dans «Italique».

3. Torquato Tasso, Il Malpiglio secondo overo del fuggir la moltitudine, in Dialoghi, a c. di G. Baffetti, Milano, Rizzoli, 1998, p. 623.

4. Sur l'importance de l'image du «studio» et de la bibliothèque dans l'œuvre en prose du Tasse cf. G. Baldassarri, La prosa de Tasso e l'universo del sapere, in AA. VV., Torquato Tasso e la cultura estense, a c. di G. Venturi, Firenze, Olschki, 1999, pp. 361-409, en particulier les pp. 36r-66.

5. Le recueil comprend le De mysteriis Aegyptiorum de Jamblique et des écrits de Porphyre, Sinesios, Psellos, Alcinoos, Speusippe, Pythagore, ainsi que le De voluptate de Ficin. Il est cité dès sa parution dans une lettre latine de l'Arioste à Alde Manuce (Ludovico Ariosto, Lettere, I, in Satire, Erbolato, Lettere, a c. di C. Segre, G. Ronchi, A. Stella, Milano, Mondadori, 1984, p. I 3 I) et est imprimé au moins sept fois au cours du XVI ${ }^{\mathrm{e}}$ siècle (M. Lowry, The World of Aldus Manutius. Business and Scholarship in Renaissance Venice, Oxford, Blackwell, I979, p. I I 5). Le Tasse en posséda deux exemplaires, qu'il lut et annota à deux moments différents de sa vie: cf. A. M. Carini, I postillati barberiniani di Torquato Tasso, «Studi Tassiani», 4 (1962), pp. 98-1 Io, et les importantes précisions de G. Baldassarri, Notizie di postillati tassiani, «Studi Tassiani», 47 (1999), pp. I17-27, pp. I 20-21. Il existe de ce recueil une réproduction moderne (Iamblichus, De mysteriis aegyptiorum. Sammelband neuplatonischer Schriften übersehen und herausgegeben von Marsilius Ficinus, Frankfurt-Main, Minerva 1972), d'où seront tirées nos citations.

6. Proclus, De anima et daemone, in Iamblichus, De mysteriis aegyptiorum, p. Io9. Le passage traduit, en abrégeant considérablement, In Alcibiadem 245.4-246.I 3 (Sur le premier Alcibiade de Platon, texte établi et traduit par A. Ph. Segonds, Paris, Les Belles Lettres, I986, pp. 293-94), qui est à son tour un long développement à partir de PLAton, Alcibiade I Io D 5 - E $\mathrm{I}$.

7. Proclus, De anima et daemone, p. I Io (In Alcibiadem 246.1 3-16; Sur le premier Alcibiade cit., p. 294).

8. TAsso, Il Malpiglio secondo, pp. 66о-6г. L'image du lien (vinculum) qui assure l'unité des sciences inspire, dans le dialogue du Tasse, une série de variations métaphoriques: «Laonde è un certo numero de le scienze che si posson legare con un legame, il quale è più saldo e di maggior prezzo che non son le catene di diamante»; «Troppo buon maestro sarebbe colui che m'isegnasse di far così preziosa catena»; «le scienze ancora son molte, e si congiunge l'una con l'altra in quella guisa che fanno gli anelli de la catena» (p. 629). L'importance de cette métaphore dans l'œuvre du Tasse est soulignée par F. Pignatti, Dalla "diversità de le vie per ogni parte infinite" alla "catena adamantina": linguaggio e retorica nei "Dialoghi" di Tasso, in AA. VV., Studi in onore di B.T. Sozzi, a c. di A. Agazzi, Bergamo, Centro di Studi Tassiani, 1991, pp. 23-35.

9. Proclus, De anima et daemone, p. I Io (In Alcibiadem 246.i 8-247.5; Sur le premier Alcibiade, p. 294).

Io. TAsso, Il Malpiglio secondo, p. 662. 
I I. Cf. au moins G. Aquilecchia, Dialoghi tassiani e dialoghi bruniani: per una comparazione delle fonti et La prosa del Tasso tra moduli attici e mediazione umanistica, in Nuove schede di italianistica, Roma, Salerno, 1994, pp. 226-39 et 353-65; G. Baldassarri, Fra "Dialogo" e "Nocturnales adnotationes": prolegomeni alla lettura del Messaggiero, «Rassegna della Letteratura Italiana», LXXVII (1972), pp. 265-93.

I 2. Il suffit de penser à une des premières octaves de la Jérusalem délivrée où l'auteur se présente comme un «peregrino» que la protection de son seigneur sauve de la fureur de la mer et conduit à bon port: «Tu, magnanino Alfonso, il qual ritogli / al furor di fortuna e guidi in porto / me peregrino errante, e fra gli scogli / e fra l'ode agitato e quasi absorto, / queste mie carte in lieta fronte accogli» (Gerusalemme liberata, I, 4). Cf. S. Zatti, Tasso contro Ariosto?, in L'ombra de Tasso. Epica e romanzo nel Cinquecento, Milano, Bruno Mondadori, i996; S. Prandi, Dal mare della Fortuna al porto della Provvidenza: appunti su un'ossessione tassiana, in AA. VV., Torquato Tasso e l'Università, a c. di W. Moretti e L. Pepe, Firenze, Olschki, 1997; G. Baldassarri, La prosa del Tasso e l'universo del sapere cit., pp. 370-72.

I 3. Une intéressante analyse formelle de cette métaphore filée est proposée par S. Bozzola, La similitudine in forma di dialogo. Prime indagini sul trattamento delle figure di analogia nei Dialoghi del Tasso, in AA. VV., Il sapere delle parole. Studî sul dialogo latino e italiano del Rinascimento, a c. di W. Geerts, A. Paternoster, F. Pignatti, Roma, Bulzoni, 200I, pp. I49-58, pp. i 5 I-52. L'importance des schémas de l'art de la mémoire dans la culture de la Renaissance est démontrée par de nombreux exemples dans le livre de L. Bolzoni, La stanza della memoria. Modelli letterari e iconografici nell'età della stampa, Torino, Einaudi, I995.

I4. TAsso, Il Malpiglio secondo, pp. 649-5 I.

I s. Cf. le commentaire qui accompagne le texte du dialogue dans l'éditions des Prose, a c. di E. Mazzali, Milano-Napoli, Ricciardi, 1959. Le Tasse lut et annota les Moralia de Plutarque, dont le De placitis philosophorum fait partie, dans une traduction latine de plusieurs auteurs (dont Erasme) publiée à Venise en I 532 (Opuscula omnia, G. A. de Sabio e fratelli). Cf. L. Chines, Tasso postillatore di Plutarco, in Torquato Tasso e l'Università cit.

I6. Pour donner une idée plus précise de cette stratégie, on reproduit ici, dans une traduction moderne, la première partie du passage de Plutarque dont le Tasse s'est inspiré pour le texte du Malpiglio cité plus haut: «Thalès fut le premier à déclarer que l'âme est par nature animée d'un mouvement perpétuel ou capable de se mouvoir elle même. Pour Pythagore, elle est un nombre qui se meut de lui-même; il substitue le terme 'nombre' à celui d'esprit'. Pour Platon, c'est une essence intelligible qui se meut d'elle même et selon un nombre harmonieux. Pour Aristote, c'est l'entéléchie première d'un corps naturel, organisé, possédant la vie en puissance: par 'entélechie' il faut entendre la puissance et l'acte. Pour Dicéarque, c'est l'harmonie des quatres éléments premiers. Pour le médecin Asclépiade, c'est l'action conjuguée des sens. Tous les auteurs énumérés ci-dessous supposent l'ame incorporelle et la définissent comme une nature capable de se mouvoir elle-même, comme une essence intelligible ou comme l'entéléchie d'un corps naturel organisé et possédant la vie en puissance. Selon les disciples d'Anaxagore, elle est semblable à l'air et c'est un corps. Selon les Stoïciens, c'est un souffle chaud. Selon Démocrite, c'est un composé enflammé, fait d'éléments qui ne peuvent être que perçus par la raison, dont la phorme est sphérique et la propriété celle du feu, ce qui signifie qu'elle est un corps»; Plutarque, Opinions des philosophes (Moralia, 898B-899A), trad. de G. Lachenaud, Paris, Les Belles Lettres, 1993.

17. L'importance des sources doxographiques (florilèges, recueil de sententiae et de loci communes) dans la culture du Tasse a été démontrée par les travaux de Bruno Basile: cf. en particulier Tasso e le Sententiae di Stobeo, «Filologia e critica», VII (I982), I, pp. I I 4-24 e La biblioteca del Tasso. Rilievi ed elenchi di libri dalle Lettere del poeta, «Filologia e critica», XXV (2000), 2-3, pp. 222-244. 


\section{Matteo Residori}

I 8. Au début du Forno ( 1578 ), par exemple, le Tasse accumule plusieurs définitions antiques de la noblesse, tirèes de Stobée, avant de choisir la meilleure en adoptant un critère rigoureux (l'exemple est cité par B. Basile, Tasso e le Sententiae di Stobeo cit.)

I9. G. Scianatico, Dallo studio di Giovanlorenzo Malpiglio, in AA. VV., Studi in onore di B.T. Sozzi, a c. di A. Agazzi, Bergamo, Centro di Studi Tassiani i991, pp. 59-70.

20. Cf. les observations de B. Basile, Sogni di terre lontane, in Poëta melancholicus. Tradizione classica e follia nell'ultimo Tasso, Pisa, Pacini, I984, pp. 325-68, en particulier les pp. 329-30.

2 I. Cf. R. H. Popkin, The History of Scepticism from Erasmus to Descartes, New York, Evanston - London, Harper and Row i 960.

22. Cf. Ch. B. Schmitt, Gianfrancesco Pico della Mirandola (1469-I533) and his critique of Aristotle, The Hague, Martinus Nijhoff i967, pp. I60-81.

23. Ioannis Franciscus Picus, Examen vanitatis doctrinae gentium et veritatis christianae disciplinae, in Opera omnia Ioannis Francisci Pici Mirandulae Domini et Concordiae Comitis, Basilaeae, ex officina Henricpetrina I 557, t. II, pp. 7 I0-I 264, p. 767. On peut aussi remarquer la proximité entre la phrase de transition qui introduit le passage de Pic («Iam nec maior in ipsa rei natura et essentia quam in numero et ordine consensus apparet») et celle qui introduisait le passage cité du Malpiglio («Né minor discordia è ne l'anima di quel che sia ne la generazione»). Il est aussi intéressant de constater à quel point la longue section sceptique de l'Apologie de Montaigne (que le Tasse ne pouvait évidemment pas connaitre) est parfois proche du Malpiglio, sans doute à cause de l'identité des sources. Voici par exemple le passage concernant les théories de l'âme: «Or voyons ce que l'humaine raison nous a appris de soi et de l'ame; non de l'ame en general, de la quelle quasi toute la philosophie rend les corps celestes et les premiers corps participants; ny de celle que Thales attribuoit aux choses mesmes qu'on tient inanimées, convié par la consideration de l'aimant, mais de celle qui nous appartient, que nous devons mieux cognoistre. [...] A Crates et Dicæarchus, qu'il n'y en avoit du tout point, mais que le corps s'esbranloit ainsi d'un mouvement naturel; à Platon, que c'estoit une substance se mouvant de soy-mesme; à Thales, une nature sans repos; à Asclepiades, une exercitation des sens; à Hesiodus et Anaximander, chose composée de terre et d'eau; à Parmenides, de terre et de feu; à Empedocles, du sang [...] à Possidonius, Cleantes et Galen, une chaleur ou complexion chaleureuse [...]» (Michel DE Montaigne, Apologie de Raimond Sebond, in Essais, a c. di P. Villey, Paris, Presses Universitaires de France, I965, II, xiI, pp. 542-43).

24. TAsso, Il Malpiglio secondo, p. 663.

25. Montaigne, Apologie de Raimond Sebond, p. 492. 